\title{
Local optical density of states in finite crystals of plane scatterers
}

\author{
Martijn Wubs*,† and A. Lagendijk ${ }^{\dagger}$ \\ Van der Waals-Zeeman Instituut, Universiteit van Amsterdam, Valckenierstraat 65, 1018 XE Amsterdam, The Netherlands
}

(Received 20 November 2001; published 2 April 2002)

\begin{abstract}
Spontaneous emission rates in a dielectric structure are position dependent and proportional to the local optical density of states. The latter can be calculated when the Green function is known. In our scalar formalism, we model dielectric slabs as infinitely thin planes and use the $T$-matrix formalism of multiplescattering theory to find an exact analytical expression for the Green function of a dielectric mirror of arbitrary number of unit cells $N$. All the propagating and guided modes of the structure are found. There are at most $N$ guided modes and their dispersion relations are studied. The guided modes appear around frequencies corresponding to the first stop band in normal direction. Local densities of propagating and guided modes are presented, also for frequencies where the layered structure acts as an omnidirectional mirror.
\end{abstract}

DOI: 10.1103/PhysRevE.65.046612 PACS number(s): 41.20.Jb, 42.70.Qs, 78.66. - w, 42.50. $-\mathrm{p}$

\section{INTRODUCTION}

Photonic crystals with a complete photonic band gap have a frequency interval in the optical regime in which the total density of states is zero [1]. In order to have such materials in which spontaneous emission in all directions would be completely suppressed, much effort is made nowadays to fabricate photonic crystals with periodic spatial variation of the refractive index in three dimensions $[2,3]$. The refractive index contrast must be high, the volume fraction of the highindex material should be small [4], the crystals preferably should have many unit cells in all three directions, and light absorption should be absent [5]. In the optical domain, these combined requirements prove to be tough, and many research groups are working on it.

In inhomogeneous materials such as photonic crystals, spontaneous emission rates, in general, are position dependent and proportional to the local density of states (LDOS) $[6,7]$. It is therefore interesting to calculate the LDOS for specific dielectric geometries as, for instance, recently was done for photonic crystals in two dimensions consisting of a finite number of cylinders [8]. Also the theoretical and experimental study of the LDOS in spherical microcavities can be mentioned here [9]. The calculation of local densities of states in infinite three-dimensional photonic crystals are numerically very involved $[10,11]$. In practice, photonic crystals have a finite size, but the LDOS of a finite crystal would be even harder to calculate. In principle, the LDOS could directly be inferred from the Green function of a photonic crystal, but Green functions are hard or practically impossible to find for finite crystals with refractive-index variations in all three dimensions. Therefore, it is interesting to look for other finite photonic systems for which Green functions can be calculated explicitly. In this paper we consider three-dimensional light propagation in a system of periodic dielectric layers, where the refractive index varies in one

\footnotetext{
*Email address: c.m.wubs@tn.utwente.nl

${ }^{\dagger}$ Present address: Complex Photonic Systems Group, Faculty of Applied Physics, University of Twente, P.O. Box 217, NL-7500 AE, The Netherlands.
}

spatial direction only. Our focus will be on the local mode densities in and around the structure. We look for a model that is simple enough to allow for analytical solutions yet realistic enough as to give insight.

Before introducing our model, we briefly discuss some of the relevant work on layered photonic crystals. Optical properties of dielectric slab structures have been studied intensively $[12,13]$, both theoretically and experimentally in pure and applied optics $[14,15]$. An optically excited atom inside a slab structure can spontaneously emit its photon into a radiative or a guided mode and both types of decay channels contribute to the total spontaneous emission rate [16]. Layered dielectric structures that recently enjoyed some interest are the so-called "omnidirectional mirrors" [17]. With the future photonic band-gap crystals they share the property of omnidirectional reflection of light in a well-defined frequency interval. Unlike inside a photonic band-gap crystal, inside layered omnidirectional mirrors the spontaneous emission rates do not go to zero [18]: in the frequency interval where light cannot enter the structure, spontaneous emission is taken over by the guided modes. Evidently, reflection experiments alone are not enough to tell what is going on inside a photonic crystal.

The perfect periodicity of infinite crystals increases the chances for analytical solutions. Analytical properties of finite crystals consisting of $N$ unit cells are more difficult to find. Fortunately it is known from transfer matrix techniques [12] how to express transmission and reflection of $N$-period Bragg mirrors simply in terms of the number $N$ and the reflection $\rho$ of a single unit cell [13]. In Ref. [19] this result was generalized to nonabsorbing layered structures where the unit cell has an arbitrary refractive-index profile. These analytical results are important. But as we learnt from the omnidirectional mirror, the knowledge of transmission and reflection is not enough for understanding spontaneous emission rates of internal sources: we also need to find the guided modes of finite one-dimensional photonic crystals consisting of an arbitrary number of unit cells. In [20] a general method was derived to compute propagating and guided modes and Green functions of multilayer dielectrics with constant refractive indices in each layer. One has to be very careful not to miss any of the guided modes. The method involves a 
numerical step, namely, the solution of boundary conditions at each dielectric interface. In our calculations, we want to avoid such a step.

In the theory of (metallic) diffraction gratings, it proved useful to study the limit of infinitely thin gratings [21] and there homogeneous layers are called impedance sheets. We study a Kronig-Penney model of a finite layered photonic crystal. Such models have been used before in the study of infinite layered photonic crystals in one [22] and two [4,23] spatial dimensions; in the latter paper also guided modes of the infinite crystal are considered. Our model differs from these works in that we consider finite crystals in three spatial dimensions, in a formalism that is particularly suited for finding all the optical modes in a unified way; the (local) density of states strongly depends on the number of spatial dimensions considered [11].

We use the $T$-matrix formalism of multiple-scattering theory (see the classics [24,25] or [26] for a recent account.) An advantage of multiple-scattering theory is that the properties of systems consisting of many scatterers can be given in terms of the properties of the individual scatterers. Boundary conditions are automatically satisfied if they are satisfied by the single scatterer. Another advantage is that the formalism simplifies the calculation of Green functions enormously. The concept of a point scatterer has proved extremely useful for random multiple scattering $[27,28]$. Band structures and local densities of states of crystals consisting of point scatterers have also been studied [6,29]. Here we propose the planar analogue of the point scatterer, the infinitely thin plane, which we coin the plane scatterer.

The organization of the paper is as follows. In Sec. II we briefly introduce the $T$-matrix formalism that we then apply to a single-plane scatterer (Sec. II A) and to $N$-plane scatterers at arbitrary positions (Sec. II B). An analytical expression for the $T$ matrix is derived in the special case that the $N$ planes are at regular distances. The specific model for a plane scatterer that will be used throughout this paper is introduced in Sec. IIC. The optical modes of the crystal of plane scatterers are presented in Sec. III and both the propagating (Sec. III A) and the guided modes (Sec. III B) are discussed. In Sec. IV the local densities of states corresponding to both propagating modes and guided modes are presented. In Sec. V we conclude.

\section{T-MATRIX FORMALISM FOR PLANE SCATTERERS}

\section{A. General properties of the single-plane scatterer}

The modification of an incoming wave $\left|\psi_{\text {in }}(\omega)\right\rangle$ into $|\psi(\omega)\rangle$ due to the presence of a potential $V(\omega)$ is given by the Lippmann-Schwinger equation

$$
|\psi(\omega)\rangle=\left|\psi_{\text {in }}(\omega)\right\rangle+g_{0}(\omega) V(\omega)|\psi(\omega)\rangle .
$$

Here $g_{0}(\omega)$ is the Green function of the system without the potential. We use the abstract bra-ket notation; later we turn to a specific representation of the waves. The field $|\psi(\omega)\rangle$ is the implicit solution of the scattering problem. We would know this field explicitly if we would know the transition matrix or $T$ matrix of the problem, which is defined via [26]

$$
|\psi(\omega)\rangle=\left|\psi_{\text {in }}(\omega)\right\rangle+g_{0}(\omega) T(\omega)\left|\psi_{\text {in }}(\omega)\right\rangle .
$$

The $T$-matrix formalism should not be confused with the transfer matrix approach, which in the study of layered systems is most commonly used $[12,13]$. Formally, one can write $T(\omega)=V(\omega)\left[1-g_{0}(\omega) V(\omega)\right]^{-1}$. The scattering problem is solved once an explicit form of the inverse operator is found. The same $T$ matrix that solves the scattering problem for the field, also solves the scattering problem (that is the Dyson-Schwinger equation) for the Green function,

$$
\begin{gathered}
G(\omega)=g_{0}(\omega)+g_{0}(\omega) V(\omega) G(\omega) \\
=g_{0}(\omega)+g_{0}(\omega) T(\omega) g_{0}(\omega) .
\end{gathered}
$$

Thus, the $T$ matrix directly leads to scattered fields and to the Green function.

Now consider an infinitely thin plane $z=z_{\alpha}$ that scatters light coming from $z<z_{\alpha}$. In this paper we consider scattering of scalar waves. If an incident plane wave $\left|\psi_{\text {in }}\right\rangle=|\mathbf{k}\rangle$ $=\left|\mathbf{k}_{\|}, k_{z}\right\rangle$ with $k_{z}$ real and greater than 0 scatters from the plane, the in-plane wave vector $\mathbf{k}_{\|}$is conserved. The total wave $\left|\psi_{\mathbf{k}}\right\rangle$ is called a "true mode" or "mode function" and it has an incoming, a transmitted, and a reflected part. The $T$ matrix for such a plane scatterer must have the form

$$
T_{\alpha}(\omega)=\frac{1}{(2 \pi)^{2}} \int \mathrm{d}^{2} \mathbf{k}_{\|} T\left(\mathbf{k}_{\|} ; \omega\right)\left|\mathbf{k}_{\|}, z_{\alpha}\right\rangle\left\langle z_{\alpha}, \mathbf{k}_{\|}\right|
$$

The function $T\left(\mathbf{k}_{\|} ; \omega\right)$ describes the light scattering as a function of in-plane wave vector and frequency. At the moment the function is not specified, but in Sec. II C a model will be derived for it. In the rest of the section, we express transmission and reflection properties of a single plane in terms of its $T$ matrix. Furthermore, we analyze the forms that the $T$ matrix can take if we assume that energy is conserved in the scattering process.

First we need a suitable representation for the planescattering problem. The free-space Green function $g_{0}(\omega)$ has the well-known real-space representation $g_{0}\left(\mathbf{r}, \mathbf{r}^{\prime} ; \omega\right)=$ $-\exp \left(i \omega^{+}\left|\mathbf{r}-\mathbf{r}^{\prime}\right|\right) /\left(4 \pi\left|\mathbf{r}-\mathbf{r}^{\prime}\right|\right)$ with $\omega^{+} \equiv \omega+i \eta$ so that the Green function corresponds to an outgoing spherical wave. In Fourier representation we have $g_{0}\left(\mathbf{k}, \mathbf{k}^{\prime} ; \omega\right)=(2 \pi)^{3} \delta^{3}(\mathbf{k}$ $\left.-\mathbf{k}^{\prime}\right) /\left[\left(\omega^{+} / c\right)^{2}-k^{2}\right]$. By the choice of the infinitesimally small positive part we select the causal Green function whose inverse Fourier transform gives zero for times $t<0$ [30]. In the following, Green functions are implicitly understood to be causal and the "+" notation is dropped. Now the form (5) of the plane scatterer suggests a mixed representation $\left\{\left|\mathbf{k}_{\|}, z\right\rangle\right\}$, where $\mathbf{k}_{\|} \equiv\left(k_{x}, k_{y}\right)$ is the wave vector parallel to the planes. Then the free-space Green function becomes

$$
\begin{aligned}
g_{0}\left(\mathbf{k}_{\|}, z ; \mathbf{k}_{\|}^{\prime}, z^{\prime} ; \omega\right) & \equiv\left\langle\mathbf{k}_{\|}, z\left|g_{0}(\omega)\right| \mathbf{k}_{\|}^{\prime}, z^{\prime}\right\rangle \\
& =(2 \pi)^{2} \delta^{2}\left(\mathbf{k}_{\|}-\mathbf{k}_{\|}^{\prime}\right) g_{0}\left(\mathbf{k}_{\|}, z-z^{\prime} ; \omega\right) .
\end{aligned}
$$

The function $g_{0}\left(\mathbf{k}_{\|}, z ; \omega\right)$ will also be called the Green function and it takes the form 


$$
g_{0}\left(\mathbf{k}_{\|}, z ; \omega\right)=\frac{\exp \left(i k_{z}|z|\right)}{2 i k_{z}}
$$

where $k_{z}=\sqrt{(\omega / c)^{2}-k_{\|}^{2}}$ can be real or imaginary. Using this and Eqs. (2) and (5), we find for the mode function

$$
\begin{gathered}
\psi_{\mathbf{k}}(\mathbf{r})=e^{i \mathbf{k} \cdot \mathbf{r}}\left[1+\frac{T\left(\mathbf{k}_{\|} ; \omega\right)}{2 i k_{z}}\right], \quad\left(z>z_{\alpha}\right) \\
=e^{i \mathbf{k} \cdot \mathbf{r}}+e^{i \mathbf{k}^{R} \cdot \mathbf{r}}\left[\frac{T\left(\mathbf{k}_{\|} ; \omega\right) e^{2 i k_{z} z}}{2 i k_{z}}\right], \quad\left(z<z_{\alpha}\right) .
\end{gathered}
$$

The expression between the square brackets in Eq. (8) is the transmission amplitude $\tau\left(\mathbf{k}_{\|} ; \omega\right)$ in terms of the $T$ matrix and between the brackets in Eq. (9) we have the reflection amplitude $\rho\left(\mathbf{k}_{\|} ; \omega\right)$. The reflected wave vector $\mathbf{k}^{R}$ equals $\left(\mathbf{k}_{\|}\right.$, $\left.-k_{z}\right)$. Thus, we have expressed transmission and reflection properties of a single plane in terms of its $T$ matrix.

Until now, we have not assumed that energy is conserved in the scattering process. Energy conservation puts a restriction on the form that $T\left(\mathbf{k}_{\|} ; \omega\right)$ can have, a restriction that we will now derive. Energy conservation can be quantified with the help of the energy flux density vector $\mathbf{J}[31]$,

$$
\mathbf{J}(\mathbf{r}, t)=-\alpha\left[\frac{\partial \psi^{*}(\mathbf{r}, t)}{\partial t} \boldsymbol{\nabla} \psi(\mathbf{r}, t)+\frac{\partial \psi(\mathbf{r}, t)}{\partial t} \boldsymbol{\nabla} \psi^{*}(\mathbf{r}, t)\right]
$$

where $\alpha$ is a proportionality constant depending on the choice of units. Since there is no energy transfer between true modes, the energy current density $\mathbf{J} \cdot \hat{z}$ in the $z$ direction should be the same for $z>z_{\alpha}$ and for $z<z_{\alpha}$ for every mode $\left|\psi_{\mathbf{k}}\right\rangle$ separately. In terms of the transmission and reflection, this leads to the requirement $|\rho|^{2}+|\tau|^{2}=1$; in terms of the $T$ matrix it reads

$$
\operatorname{Im} T\left(\mathbf{k}_{\|} ; \omega\right)=-\frac{1}{2} \frac{\left|T\left(\mathbf{k}_{\|} ; \omega\right)\right|^{2}}{k_{z}} .
$$

This is the optical theorem for the plane scatterer. Note that it only holds for real $k_{z}$. The most general $T$ matrix satisfying the optical theorem has the form

$$
T\left(\mathbf{k}_{\|} ; \omega\right)=-\left[F^{-1}\left(\mathbf{k}_{\|} ; \omega\right)-i /\left(2 k_{z}\right)\right]^{-1},
$$

where the optical potential $F\left(\mathbf{k}_{\|} ; \omega\right)$ for the plane is a real function of its arguments. Furthermore, for planes that scatter isotropically, we have $F\left(\mathbf{k}_{\|} ; \omega\right)=F\left(k_{\|}, \omega\right)$. The Eqs. (9) and (12) together show that a larger optical potential leads to a higher reflectivity of the plane.

\section{B. $N$-plane scatterers}

Consider a system of $N$ identical and parallel plane scatterers at arbitrary positions $z_{\alpha}, z_{\beta}$, and so on. At first, we do not assume that energy is conserved in the scattering process, so that the single-plane $T$ matrices need not be of the form (12). Just as in the one-plane case, we want to know mode functions and Green functions of the $N$-plane system. This we do by calculating the $T$ matrix $T^{(N)}\left(\mathbf{k}_{\|} ; \omega\right)$. Formally, we have as in the one-plane case that $T^{(N)}=V\left(1-g_{0} V\right)^{-1}$. The inverse is defined as an infinite series, so that

$$
T^{(N)}=V+V g_{0} V+V g_{0} V g_{0} V+\cdots,
$$

where now $V$ is the sum of the one-plane potentials. We now try to actually do the summation of this infinite series of plane-scattering events. Then, instead of writing the $T$ matrix in terms of potentials, it is more efficient to write it in terms of the one-plane $T$ matrices,

$$
\begin{aligned}
T^{(N)}= & \sum_{\alpha} T_{\alpha}+\sum_{\beta \neq \alpha} \sum_{\alpha} T_{\beta} g_{0} T_{\alpha} \\
& +\sum_{\gamma \neq \beta} \sum_{\beta \neq \alpha} \sum_{\alpha} T_{\gamma} g_{0} T_{\beta} g_{0} T_{\alpha}+\cdots
\end{aligned}
$$

Written out explicitly, the term of third order in the singleplane $T$ matrices in this series is

$$
\begin{aligned}
& \frac{1}{(2 \pi)^{2}} \sum_{\alpha} \sum_{\beta} \sum_{\gamma} \int \mathrm{d}^{2} \mathbf{k}_{\|}\left|\mathbf{k}_{\|}\right\rangle\left\langle\mathbf{k}_{\|}\right| T^{3}\left(\mathbf{k}_{\|} ; \omega\right) g_{0}\left(\mathbf{k}_{\|}, z_{\alpha}\right. \\
& \left.\quad-z_{\beta} ; \omega\right)\left(1-\delta_{\alpha \beta}\right) g_{0}\left(\mathbf{k}_{\|}, z_{\beta}-z_{\gamma} ; \omega\right) \\
& \quad \times\left(1-\delta_{\beta \gamma}\right)\left|z_{\alpha}\right\rangle\left\langle z_{\gamma}\right| .
\end{aligned}
$$

Now define the matrix $D_{\alpha \beta}\left(\mathbf{k}_{\|} ; \omega\right) \equiv g_{0}\left(\mathbf{k}_{\|}, z_{\alpha}-z_{\beta} ; \omega\right)(1$ $-\delta_{\alpha \beta}$ ) and notice that the third-order term can be rewritten as

$$
\begin{aligned}
& \frac{1}{(2 \pi)^{2}} \sum_{\alpha} \sum_{\beta} \int \mathrm{d}^{2} \mathbf{k}_{\|}\left|\mathbf{k}_{\|}\right\rangle\left\langle\mathbf{k}_{\|}\right| T\left(\mathbf{k}_{\|} ; \omega\right) \\
& \quad \times\left\{\left[T\left(\mathbf{k}_{\|} ; \omega\right) \mathbf{D}\left(\mathbf{k}_{\|} ; \omega\right)\right]^{2}\right\}_{\alpha \beta}\left|z_{\alpha}\right\rangle\left\langle z_{\beta}\right| .
\end{aligned}
$$

So we can see the square of the matrix (TD) in the thirdorder terms. The fourth-order terms feature the cube of the matrix (TD), and so on. This matrix structure enables us to sum the infinite series exactly: the $T$ matrix of $N$ parallel and identical plane scatterers is given by

$$
\begin{aligned}
T^{(N)}(\omega)= & \frac{1}{(2 \pi)^{2}} \sum_{\alpha} \sum_{\beta} \int \mathrm{d}^{2} \mathbf{k}_{\|} \sum_{\alpha, \beta}\left|\mathbf{k}_{\|}, z_{\alpha}\right\rangle T_{\alpha \beta}^{(N)}\left(\mathbf{k}_{\|}, \omega\right) \\
& \times\left\langle z_{\beta}, \mathbf{k}_{\|}\right|
\end{aligned}
$$

where

$$
T_{\alpha \beta}^{(N)}\left(\mathbf{k}_{\|}, \omega\right)=T\left(\mathbf{k}_{\|} ; \omega\right)\left[1-T\left(\mathbf{k}_{\|} ; \omega\right) \mathbf{D}\left(\mathbf{k}_{\|} ; \omega\right)\right]_{\alpha \beta}^{-1} .
$$

The problem of finding the $T$ matrix is now reduced to the much simpler problem of finding the inverse of the $N \times N$ matrix $T^{(N)}$. For arbitrary positions of the planes, the inversion must be done numerically. The matrix elements depend on the positions and effective thickness of the parallel planes, and on the frequency and in-plane wave vector of the light. Since Eq. (17) is valid for arbitrary positions of the plane scatterers, it could be the starting point for studying random scattering. 
The important special case that will be studied here is the situation where the $N$ planes are positioned at regular distances $a$ from each other, at positions $z_{1}=a, z_{2}$ $=2 a, \ldots, z_{N}=N a$. We then have a three-dimensional optical Kronig-Penney model for a finite photonic crystal with periodic variation of the refractive index in one dimension. The regular distances between the planes enable us to do the inversion of the matrix in Eq. (18) analytically, as we shall discuss now.
Define $\mu \equiv-2 i k_{z} / T\left(\mathbf{k}_{\|}, \omega\right)$ and $x \equiv \exp \left(i k_{z} a\right)$. Then $T_{\alpha \beta}^{(N)}$ in Eq. (18) can be rewritten as $-2 i k_{z} M_{\alpha \beta}^{-1}$ with

$$
M_{k l} \equiv \mu \delta_{k l}+\left(1-\delta_{k l}\right) x^{|k-l|}
$$

We should like to know the inverse of this $N \times N$ matrix $\mathbf{M}$ for arbitrary $N$ and in the Appendix this mathematical problem is solved. The result is

$$
\begin{aligned}
T_{\alpha \beta}^{(N)}= & \frac{-2 i k_{z}}{(2-\mu) x^{2}}\left\{h_{N}(\alpha, \beta)-x h_{N}(\alpha+1, \beta)-x h_{N}(\alpha, \beta+1)+x^{2} h_{N}(\alpha+1, \beta+1)\right. \\
& \left.+\frac{\left[x h_{N}(\alpha+1,1)-h_{N}(\alpha, 1)\right]\left[h_{N}(1, \beta)-x h_{N}(1, \beta+1)\right]}{1+h_{N}(1,1)}\right\},
\end{aligned}
$$

where the function $h_{N}(\alpha, \beta)$ is defined as

$$
h_{N}(\alpha, \beta) \equiv \frac{(2-\mu) x}{2(1-\mu)} \frac{1}{N+1} \sum_{m=1}^{N}\left\{\frac{\cos [(\alpha+\beta) m \pi /(N+1)]-\cos [(\alpha-\beta) m \pi /(N+1)]}{C-\cos [m \pi /(N+1)]}\right\} .
$$

From the definition of $h_{N}$ it follows that $\mathbf{M}^{-1}$ is a symmetric matrix, as is $\mathbf{M}$. The matrix elements of $T^{(N)}$ strongly depend on the form of the constant $C$ in Eq. (21) and for real $k_{z}$ it is given by

$$
C=\frac{2 x^{2}-\mu\left(1+x^{2}\right)}{2 x(1-\mu)}=\cos \left(k_{z} a\right)+\sin \left(k_{z} a\right)\left[\frac{2 k_{z} \operatorname{Re}(T)+i\left(2 k_{z} \operatorname{Im}(T)+|T|^{2}\right)}{4 k_{z}^{2}+|T|^{2}+4 k_{z} \operatorname{Im}(T)}\right] .
$$

Fortunately, the summation in Eq. (21) can be done exactly so that we end up with a simpler expression for $h_{N}$ [32],

$$
h_{N}(\alpha, \beta)=\frac{(2-\mu) x}{2(1-\mu)}\left\{\frac{\cos [(N+1-|\alpha-\beta|) K a]-\cos [(N+1-(\alpha+\beta)) K a]}{\sin [K a] \sin [(N+1) K a]}\right\}
$$

where the constant $K=K\left(\mathbf{k}_{\|} ; \omega\right)$ is defined by $K a$ $=\arccos (C)$. (The arccosine is a multiple-valued function but the function $h_{N}$ is not; for numerical evaluation of $h_{N}$ the Chebyshev polynomials are useful.) Evidently, $K$ has dimension $\left[\mathrm{m}^{-1}\right]$ and in fact, it is equal to the Bloch wave vector of an infinite crystal of plane scatterers separated by a distance $a$. In general, $K$ is complex. The important point that the Bloch wave vector that is normally associated with the infinite periodic potential also plays a role in the analysis of a finite crystal, was also stressed in Ref. [19]. Here the Bloch wave vector shows up in an analytical expression for the $T$ matrix of a finite crystal of arbitrary size $N$. No restrictions on the form of the single-plane $T$ matrix $T\left(\mathbf{k}_{\|} ; \omega\right)$ have been applied in the derivation.

Let us now assume that energy is conserved. This is the case when every single plane has a $T$ matrix that satisfies the optical theorem (12). Then it is evident from Eq. (22) that the constant $C$ becomes real. Moreover, when the optical theorem holds, a simpler expression for $C$ can be found by inserting for the single-plane $T$ matrix the form (12)

$$
C=\cos \left(k_{z} a\right)-\left(\frac{F\left(\mathbf{k}_{\|} ; \omega\right)}{2 k_{z}}\right) \sin \left(k_{z} a\right) \text {. }
$$

One can see that without energy dissipation, the Bloch wave vector is either real (when $-1 \leqslant C \leqslant 1$ ) or purely imaginary (otherwise). Imaginary Bloch wave vectors $K\left(\mathbf{k}_{\|}, \omega\right)$ correspond to transmission stop band regions in $\left(\mathbf{k}_{\|}, \omega\right)$ space. This will be illustrated in Sec. III A.

\section{Model for the single-plane scatterer}

In the preceding subsection, the $T$ matrix of the singleplane scatterer was assumed to be known. Now we will derive a model for it, starting from a potential in the wave equation. Consider a dielectric slab of thickness $d$ and dielectric function $\varepsilon$. In principle, the dielectric function is a frequency-dependent complex function [33], but here we neglect dispersion and loss altogether by choosing $\varepsilon$ real and constant. Choose the $z$ axis such that it is perpendicular to the surfaces, defining the latter as the surfaces $z=z_{\alpha}$ and $z$ $=z_{\alpha}+d$. Then the scalar wave equation is

$$
\left[\frac{\varepsilon(\mathbf{r})}{c^{2}} \frac{\partial^{2}}{\partial t^{2}}-\nabla^{2}\right] \psi(\mathbf{r} ; t)=0
$$


where $\varepsilon(\mathbf{r})=\varepsilon$ for $z$ between $z_{\alpha}$ and $z_{\alpha}+d$, and $\varepsilon(\mathbf{r})=1$ elsewhere. The Maxwell wave equations for the electric and magnetic fields [12] reduce to this scalar wave equation in some special cases: Eq. (25) describes the electric field of s-polarized light or the magnetic field of p-polarized light for layered dielectric media. Suppose now that the thickness of the slab is much smaller than the wavelengths of light we are interested in. Then we can model the slab as a plane scatterer. We define the product of slab thickness $d$ and polarizability $\varepsilon-1$ as the effective thickness $D_{\text {eff }}$ of the plane scatterer. We find the single-plane $T$ matrix by solving the associated equation for the Green function in frequency space,

$$
\left[\nabla_{r}^{2}+(\omega / c)^{2}\right] G\left(\mathbf{r}, \mathbf{r}^{\prime} ; \omega\right)=\delta^{3}\left(\mathbf{r}-\mathbf{r}^{\prime}\right)+V(\mathbf{r}, \omega) G\left(\mathbf{r}, \mathbf{r}^{\prime} ; \omega\right)
$$

with $V(\mathbf{r}, \omega)=-(\omega / c)^{2} D_{\text {eff }} \delta\left(z-z_{\alpha}\right)$. It is easy to check that a solution of the Dyson-Schwinger equation (3) is a solution of the equation above, provided that in Eq. (3) we take the potential $V(\omega)=-D_{\text {eff }}(\omega / c)^{2}\left|z_{\alpha}\right\rangle\left\langle z_{\alpha}\right|$. We shall now solve the Dyson-Schwinger equation, which in the mixed representation becomes

$$
\begin{aligned}
\left\langle\mathbf{k}_{\|}, z|G(\omega)| \mathbf{k}_{\|}^{\prime}, z^{\prime}\right\rangle= & (2 \pi)^{2} \delta\left(\mathbf{k}_{\|}-\mathbf{k}_{\|}^{\prime}\right) g_{0}\left(\mathbf{k}_{\|}, z-z^{\prime} ; \omega\right) \\
& +g_{0}\left(\mathbf{k}_{\|}, z-z_{\alpha} ; \omega\right)\left[-D_{\text {eff }}(\omega / c)^{2}\right] \\
& \times\left\langle\mathbf{k}_{\|}, z_{\alpha}|G(\omega)| \mathbf{k}_{\|}^{\prime}, z^{\prime}\right\rangle .
\end{aligned}
$$

If in this equation we put $z$ equal to $z_{\alpha}$, then we can solve the equation for $\left\langle\mathbf{k}_{\|}, z_{\alpha}|G(\omega)| \mathbf{k}_{\|}^{\prime}, z^{\prime}\right\rangle$. If we insert this particular solution back into the Eq. (27) for general $z$, then we can also find the Green function for general $z$, $\left\langle\mathbf{k}_{\|}, z|G(\omega)| \mathbf{k}_{\|}^{\prime}, z^{\prime}\right\rangle=(2 \pi)^{2} \delta\left(\mathbf{k}_{\|}-\mathbf{k}_{\|}^{\prime}\right) G\left(\mathbf{k}_{\|} ; z, z^{\prime} ; \omega\right)$ with

$$
\begin{aligned}
G\left(\mathbf{k}_{\|} ; z, z^{\prime} ; \omega\right)= & g_{0}\left(\mathbf{k}_{\|} ; z-z^{\prime} ; \omega\right)+g_{0}\left(\mathbf{k}_{\|} ; z-z_{\alpha} ; \omega\right) \\
& \times T\left(\mathbf{k}_{\|} ; \omega\right) g_{0}\left(\mathbf{k}_{\|} ; z_{\alpha}-z^{\prime} ; \omega\right),
\end{aligned}
$$

where the frequency- and wave-vector-dependent $T$ matrix equals

$$
\begin{aligned}
T\left(\mathbf{k}_{\|} ; \omega\right) & =\frac{-D_{\text {eff }}(\omega / c)^{2}}{1+g_{0}\left(\mathbf{k}_{\|}, 0 ; \omega\right) D_{\text {eff }}(\omega / c)^{2}} \\
& =-\left\{\left[(\omega / c)^{2} D_{\text {eff }}\right]^{-1}-i /\left(2 k_{z}\right)\right\}^{-1} .
\end{aligned}
$$

This $T$ matrix is of the form (12) and hence it satisfies the optical theorem (11). Note that $T$ depends on $k_{\|}$rather than on $\mathbf{k}_{\|}$: the optical potential $F\left(\mathbf{k}_{\|} ; \omega\right)$ equals $(\omega / c)^{2} D_{\text {eff }}$ in this model, so that it depends only on frequency and not on the magnitude or direction of the wave vector of the incoming light. A $T$ matrix of a point scatterer can only be defined after a careful regularization of the free-space Green function $g_{0}\left(\mathbf{r}, \mathbf{r}^{\prime} ; \omega\right)$ because the latter diverges in the limit $\left|\mathbf{r}-\mathbf{r}^{\prime}\right|$ $\rightarrow 0$ [28]. Here, in the $T$ matrix for plane scatterers (29) it is the free-space Green function $g_{0}\left(\mathbf{k}_{\|}, z-z^{\prime} ; \omega\right)$ that appears

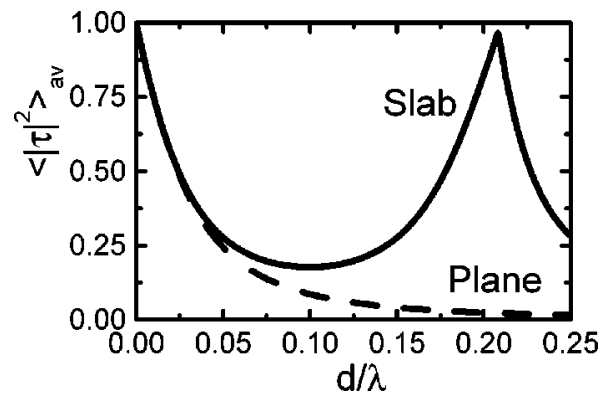

FIG. 1. Solid line: angle-averaged transmission through a slab of refractive index $\mathrm{n}=2.6$ and thickness $d$, as a function $d / \lambda$. Dashed line: angle-averaged transmission through a plane scatterer with effective thickness $D_{\text {eff }}=\left(n^{2}-1\right) d$, as a function of $d / \lambda$.

in the limit of $\left|z-z^{\prime}\right| \rightarrow 0$. Fortunately, there is no divergence so that we do not need a regularization procedure for plane scatterers.

Now that we have a one-parameter model for the $T$ matrix of a plane, we can use Eqs. (8) and (9) to find the transmitted and reflected amplitudes $\tau$ and $\rho$. The transmission amplitude is

$$
\tau\left(\mathbf{k}_{\|} ; \omega\right)=\frac{1}{1-i D_{\mathrm{eff}}(\omega / c)^{2} /\left(2 k_{z}\right)} .
$$

Clearly, transmitted intensities range from one to zero when the effective thickness goes from zero to infinity.

In the remaining section, we elaborate on the question how well slabs of finite thickness can be modeled as plane scatterers. We compare both cases by looking at the average transmission, with the average taken over $4 \pi$ solid angle. For the plane scatterer the average transmission can be calculated directly from Eq. (30), giving

$$
\left\langle|\tau|^{2}\right\rangle_{\mathrm{av}}=1-\left(\pi D_{\mathrm{eff}} / \lambda\right) \arctan \left[\lambda /\left(\pi D_{\mathrm{eff}}\right)\right] .
$$

Light transmission through a finite slab is described by Airy's formulas [12] and the angle average must be calculated numerically. Figure 1 shows the average transmission (for $s$-polarized light) of a $\mathrm{TiO}_{2}$ slab of thickness $d$ in air as well as the average transmission of scalar waves through a plane scatterer with effective thickness $D_{\text {eff }}=\left(n_{\mathrm{TiO}_{2}}^{2}-1\right) d$. The transmissions are plotted as a function of thickness $d$ divided by the wavelength $\lambda \equiv 2 \pi c / \omega$; the refractive index $n_{\mathrm{TiO}_{2}}$ is 2.6. Figure 1 shows that slabs thinner than $\lambda / 20$ can be modeled adequately as plane scatterers with effective thickness $D_{\text {eff }}=\left(n^{2}-1\right) d$. Thicker slabs show resonances in the average transmission (due to interference effects) that the planescattering model does not describe.

To give a concrete example, we consider a $d=26-\mathrm{nm}$ thick slab of $\mathrm{TiO}_{2}$. If one constructs a Bragg mirror of such slabs alternating with air with a period of $260 \mathrm{~nm}$, then evidently $10 \%$ of the Bragg mirror consists of $\mathrm{TiO}_{2}$, which is also the typical number for three-dimensional photonic airsphere crystals [34]. In normal direction the optical path length of the unit cell is $300 \mathrm{~nm}$, so that the Bragg mirror has its first Bragg reflection at around $600 \mathrm{~nm}$ in that direction. 


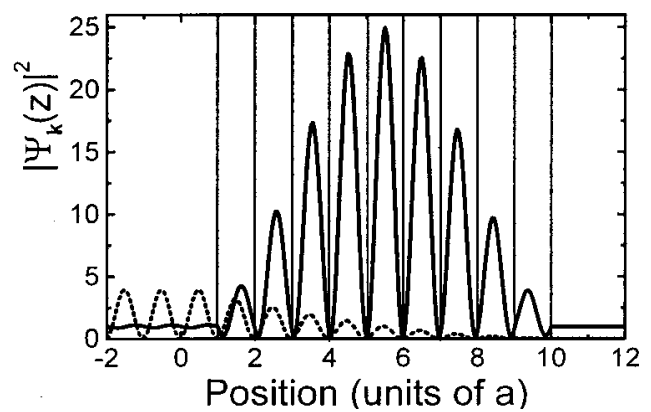

FIG. 2. Absolute values squared of two mode functions of a system of $N=10$ plane scatterers, as a function of the $z$ position in the structure. The dotted line corresponds to a mode with $a / \lambda$ $=0.50$ and the solid line to a mode with $a / \lambda=0.51$. Modes correspond to incoming plane waves from $z=-\infty$ in the direction normal to the planes. The effective thickness of the plane scatterers is $D_{\text {eff }}=0.46 a$.

At that wavelength we have $d=0.043 \lambda$, so that the single slab can indeed be described as a plane scatterer (and the Bragg mirror as a periodic array of plane scatterers). Our choice of $D_{\text {eff }}$ proceeds only a bit different: we choose $D_{\text {eff }}$ $=0.23 \lambda_{0}$, so that for wavelengths around $\lambda_{0}=600 \mathrm{~nm}$ the plane scatterer has the same average transmission of $32 \%$ as the slab. Furthermore, we choose the distance $a$ between the planes to be $300 \mathrm{~nm}$, so that the (blue edge of the) stop band in normal direction will be at $\lambda_{0}=600 \mathrm{~nm}$.

In our scalar formalism we arrive at the same model for transmission and reflection of an infinitely thin plane as was found in Ref. [23] in another formalism, for s-polarized light. There, the authors start from a boundary condition analysis of the Maxwell fields around a dielectric slab in the limits $\varepsilon \rightarrow \infty$ and $d \rightarrow 0$ while keeping the product $m=\varepsilon d$ constant. From our calculations and Fig. 1, it follows that it is actually the product $D_{\text {eff }}=(\varepsilon-1) d$ that should be kept constant while taking the limits.

\section{OPTICAL MODES}

\section{A. Propagating modes}

With the $T$ matrix in hand, in Eq. (20), we can use the solution (2) of the Lippmann-Schwinger equation to calculate the propagating modes of the finite photonic crystal. The propagating modes in our model are labeled by the wave vector and frequency of the corresponding incoming plane wave; actually they are related through $(\omega / c)^{2}=k^{2}$. The propagating mode $\left|\psi_{\mathbf{k}}(\omega)\right\rangle$ corresponding to an $N$-plane crystal is given by

$$
\begin{aligned}
\psi_{\mathbf{k}}(\mathbf{r} ; \omega)= & e^{i \mathbf{k} \cdot \mathbf{r}}-\frac{i}{2 k_{z}} e^{i \mathbf{k} \| \cdot \mathbf{r}} \sum_{\alpha, \beta=1}^{N} \exp \left[i k_{z} \mid z\right. \\
& \left.-z_{\alpha} \mid\right] T_{\alpha \beta}^{(N)}\left(\mathbf{k}_{\|} ; \omega\right) e^{i k_{z} z_{\beta}} .
\end{aligned}
$$

If we put $N=1$ in this equation, then we find back the mode function (8) for the single plane. In Fig. 2 the absolute values squared of the mode functions are plotted for two modes propagating normal to a crystal of $N=10$ planes. (We choose

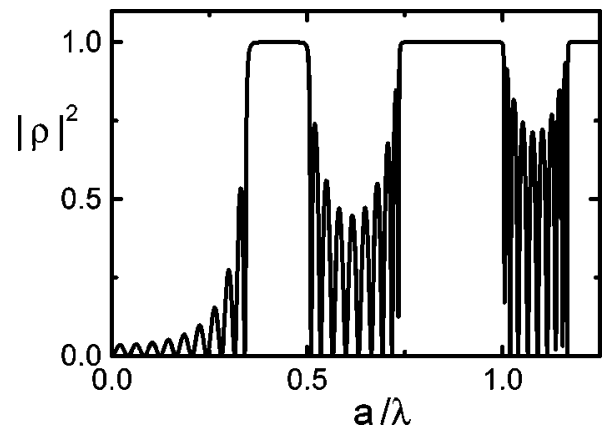

FIG. 3. Reflectivity $|\rho|^{2}$ of normally incident waves by a crystal of $N=10$ plane scatterers separated by a distance $a$, as a function of $a / \lambda$.

the normal direction, but any direction is possible.) As is clear from the figure, the slight frequency difference of the modes has an enormous influence on the mode profile in the crystal: the mode with $a / \lambda=0.5$ is almost completely reflected, as the maximum amplitude of almost 4 of the almost standing wave at the left-hand side of the structure indicates; the mode with $a / \lambda=0.51$ has an amplitude that grows inside the structure and is almost completely transmitted.

As can be seen from Eq. (32) and from Fig. 2, the absolute value squared of $\psi_{\mathbf{k}}(\mathbf{r} ; \omega)$ is a constant for $z>N a$. This is the (intensity) transmission $|\tau|^{2}$ through the $N$-plane system. Since there are no losses, reflection and transmission add up to one. In Fig. 3 the reflection is plotted for light incoming in the normal direction, as a function of frequency. The prominent features are the stop bands, the frequency intervals for which light cannot enter the crystal in this direction. The mode with $a / \lambda=0.5$ of Fig. 2 falls just inside the first stop band in Fig. 3, whereas the other mode corresponds to the first reflection minimum at the blue side of this first stop band.

The stop bands correspond to frequencies for which the Bloch wave vector [proportional to the cosine of Eq. (12)] is a complex number. In between the stop bands, the Bloch wave vector is real and there one finds the reflection maxima and minima that are characteristic for periodic media. In Fig. 3 higher-order stop bands become broader. This is because the optical potential $D_{\text {eff }}(\omega / c)^{2}$ rapidly grows with frequency since we do not take the frequency dispersion of $D_{\text {eff }}$ into account. Of course, one could improve on this by taking dispersion into account and writing $D_{\text {eff }}=[\varepsilon(\omega)-1] d$, but the optical theorem (11) only holds as long as the complex part of $\varepsilon(\omega)$ can be neglected.

Transmission and mode profiles of propagating modes in layered systems usually are calculated with the transfer matrix technique $[12,13]$. Here we showed that for a crystal of plane scatterers one can alternatively use the $T$-matrix formalism. For presentation purposes we chose $N=10$, but we also considered mode profiles and reflection for 200 planes and more planes do not form a problem: the $T$-matrix elements of $N$ planes are known analytically and the calculation of the mode function boils down to a simple summation over the $N^{2}$ matrix elements $T_{\alpha \beta}^{(N)}\left(\mathbf{k}_{\|} ; \omega\right)$. 


\section{B. Guided modes}

In the preceding section we found a continuum of propagating modes with real wave vector components in all three directions. The crystals of plane scatterers could also support a finite number of guided modes that propagate along the planes with in-plane wave vectors $k_{\|}>\omega / c$ so that $k_{z}=i \kappa$ and $k_{\|}^{2}-\kappa^{2}=(\omega / c)^{2}$. The imaginary wave vector in the $z$ direction makes that guided modes decay exponentially outside the scatterer: unlike propagating modes, guided modes do not correspond to an incident wave. It is the dispersion relations of the guided modes that we concentrate on here; the contribution of the guided modes to the local density of states is discussed in Sec. IV.

In general, the bound states of a scattering system can be found as the solutions of the Lippmann-Schwinger equation in the absence of an incident wave [26]. A guided mode $\left|\psi_{\kappa}\right\rangle$ of the crystal of plane scatterers is bound in the $z$ direction and it satisfies

$$
\psi_{\kappa}(z ; \omega)=\int_{-\infty}^{+\infty} \mathrm{d} z_{1} g_{0}\left(\mathbf{k}_{\|}, z-z_{1} ; \omega\right) V\left(z_{1} ; \omega\right) \psi_{\kappa}\left(z_{1} ; \omega\right)
$$

with the potential $V(z ; \omega)=-F \sum_{n=1}^{N} \delta(z-n a), \quad F$ $=D_{\text {eff }}(\omega / c)^{2}$, and the free-space Green function as defined in Eq. (7). Given a frequency $\omega$ and the number of planes $N$, this equation will have a finite number of solutions. In order to find them, define the $N$-dimensional vector $\boldsymbol{\Psi}$ with components $\Psi_{j}=\psi_{\kappa}\left(z_{j}\right)$ in which the $z_{j}$ are the plane positions. Using Eq. (33), the following homogeneous linear system of equations can be derived:

$$
\begin{gathered}
\sum_{\beta=1}^{N}\left[\delta_{\alpha \beta}+g_{0}\left(k_{\|}, z_{\alpha}-z_{\beta} ; \omega\right) F\right] \Psi_{\beta}=0 \text { for } \\
\alpha=1,2, \ldots, N .
\end{gathered}
$$

Nontrivial solutions of Eq. (34) only exist if the determinant of the coefficient matrix vanishes. Now this coefficient matrix can be rewritten as $-F\left(T^{(N)}\right)_{\alpha \beta}^{-1}$ with the use of the general definition of the $T$ matrix $T \equiv V\left(1-g_{0} V\right)^{-1}$. So the guided modes can be found as the poles of the $T$ matrix. This is the direct connection between the guided modes and the $T$ matrix.

The guided modes of a single plane are easy to find. For $N=1$, the $T$ matrix $T_{11}^{(1)}\left(\mathbf{k}_{\|} ; \omega\right)$ of Eq. (20) reduces to $T\left(\mathbf{k}_{\|} ; \omega\right)$ as in Eq. (29). The one-plane $T$ matrix has a pole $\kappa_{1}^{(1)}=(\omega / c)^{2} D_{\text {eff }} / 2$. This is the dispersion relation for the guided mode of a single plane. (One could equivalently present the dispersion relation as $\omega$ versus $k_{\|}$, but here and in the following, we choose to present $\omega$ versus $\kappa$.) The dispersion relation shows that for every frequency, a single plane has one and only one guided mode. The same single guided mode for a single plane was found in Ref. [23] using another formalism; the case of finite $N>1$ was not considered there.

Why does a single-plane scatterer have only one guided mode? Dielectric slabs of finite thickness can support more than one guided mode (depending on its thickness $d$ and dielectric constant $\varepsilon$ ). These guided modes other than the first one are orthogonal to this first one. Their mode functions have their extreme values inside the slab and change sign at least once inside the slab. In the limit of an infinitely thin slab, the mode functions remain continuous, so that the maxima, the minima, and the zeroes should coincide on the plane. Therefore, a plane scatterer can support at most one guided mode.

How many guided modes exist in a crystal of $N$-plane scatterers? We have just derived that they occur when the determinant $\operatorname{det}\left[\left(\mathbf{T}^{(N)}\right)^{-1}\right]$ is equal to zero. With the definition of the matrix $\mathbf{M}$ in Eq. (19) this condition simplifies to $\operatorname{det}\left[\mathbf{M}\left(k_{\|} ; \omega\right)\right]=0$. A formula for this determinant is given in Eq. (A11) of the Appendix, for an arbitrary number of planes $N$. The result is that guided modes correspond to solutions of $1+h_{N}(1,1)=0$, with $h_{N}(\alpha, \beta)$ given in Eq. (23). We assume that the planes do not absorb light and use the form (12) for the single-plane $T$ matrix. Then the condition for guides modes to exist becomes

$$
\left[2 \kappa+F\left(\mathbf{k}_{\|} ; \omega\right)\right] e^{-\kappa a} \frac{\sin (N K a)}{\sin [(N+1) K a]}=2 \kappa .
$$

This single equation determines the number of guided modes that can exist at a certain frequency for an arbitrary system size $N$. The frequency dependence of the equation is in the Bloch wave vector $K$ and in the optical potential $F$.

A new guided mode appears at a certain frequency with $\kappa=0$ and when the frequency becomes higher, this mode becomes more localized to the structure so that $\kappa$ increases. Thus, we can count guided modes at their point of first appearance, at $\kappa=0$. There we find that the condition (35) simplifies to $\sin (N K a)=0$. Now for wave vectors $k_{z}=i \kappa$, the Bloch wave vector satisfies

$$
\cos (K a)=\cosh (\kappa a)-[F /(2 \kappa)] \sinh (\kappa a),
$$

so that with $F=D_{\text {eff }}(\omega / c)^{2}$ we find guided modes with frequencies

$$
\begin{aligned}
& \omega_{m}(\kappa=0)=\sqrt{2 c^{2} /\left(a D_{\text {eff }}\right)} \sqrt{1-\cos (m \pi / N)} \\
& \text { for } m=0,1, \ldots, N-1
\end{aligned}
$$

It turns out that for systems of $N$-plane scatterers at a fixed frequency, there are at most $N$ guided modes. All $N$ guided modes emerge in the frequency interval $0 \leqslant \omega \leqslant 2 c / \sqrt{a D_{\text {eff }}}$. An increase in the effective thickness of the planes makes this frequency interval smaller, because at the same frequency the modes have larger $\kappa$ and are more localized, just as was analyzed for the single plane above. Or vice versa, for fixed $\kappa$ the frequency of a mode becomes lower when the effective thickness increases. In the limit $N \rightarrow \infty$ a band of guided modes is formed. The case of an infinite number of planes was considered in Ref. [23] and there indeed the band at $\kappa=0$ is given by the frequency interval given above.

Figure 4 shows the dispersion of the modes $\omega_{m}(\kappa)$ as a function of $\kappa$, in units of the plane separation $a$, for $N=10$. Actually, the frequencies are plotted as $a / \lambda$, and all guided 


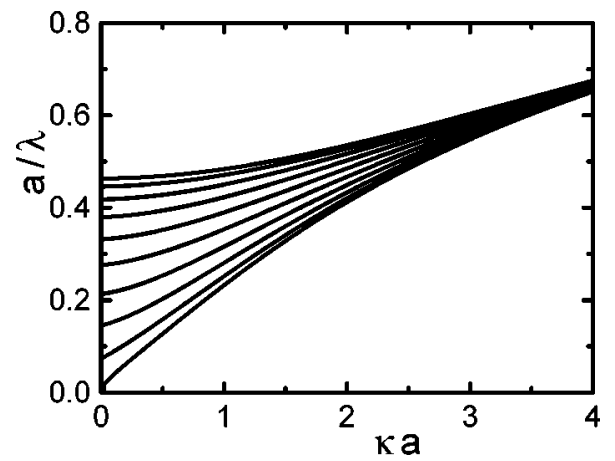

FIG. 4. Dispersion relations of the guided modes for a ten-plane system. The $\kappa$ on the horizontal axis equals $-i k_{z}$. The effective thickness of the plane scatterers is $D_{\text {eff }}=0.46 a$.

modes are indeed seen to appear at $\kappa=0$ when $0 \leqslant a / \lambda$ $\leqslant(1 / \pi) \sqrt{a / D_{\text {eff }}}=0.47$. The figure illustrates that when the product $\kappa a$ is much bigger than 1 , the planes guide light independently so that the $N$ modes all have frequencies near the one-plane frequency $\sqrt{2 \kappa / D_{\text {eff }}}$. For smaller $\kappa a$, the $N$ planes interact more strongly and the frequencies of the modes become more separated. So going from right to left in the figure, we go from $N$ independent single-plane guided modes to $N$ solutions of a genuine $N$-plane problem. The physical way to traverse the plot is of course by changing the frequency: at frequencies just below $2 c / \sqrt{a D_{\text {eff }}}$ there are both well-delocalized and well-localized modes. At higher frequencies, all guided modes are localized, whereas at lower frequencies the inverse decay lengths $(\kappa)^{-1}$ are larger so that only delocalized modes are to be expected. The localization of the guided modes will be illustrated in Sec. IV.

Now let us compare Fig. 4 with Fig. 3 that showed the stop bands for light falling on the planes in the normal direction. The first stop band in normal direction occurs for light with wavelengths $0.35 \leqslant a / \lambda \leqslant 0.50$. Light propagation normal to the planes is strongly suppressed. In this same frequency interval, the four last guided modes of the structure appear. More generally, for our choice of $D_{\text {eff }}$, all the interesting behavior of the guided modes appears around the first stop band for light propagating in the normal direction. Even when the plane crystal blocks light coming from almost all directions, then light of this same frequency can be guided inside the structure. When $D_{\text {eff }}$ is decreased, the stop bands become narrower, whereas the frequency interval in which the guided modes appear becomes wider.

\section{LOCAL DENSITY OF STATES}

In inhomogeneous dielectrics, the spontaneous emission rate of a radiating atom depends on its position and is proportional to a quantity that is often called the local density of states (LDOS). In quantum optics, many quantum states of light can be associated with what in this paper we call an optical "mode," so that the words "state" and "mode" have a different meaning. Actually, with "density of states" it is meant "density of modes" and indeed the latter term is sometimes used $[18,19]$. But we stick to the familiar term LDOS and assume that it will not confuse the reader. The
LDOS is a function of position and frequency and will be denoted by $\rho(\mathbf{r}, \omega)$. (This $\rho$ is different from the reflection amplitude of Sec. II C.)

For scalar waves in three dimensions, $\rho(\mathbf{r}, \omega)$ is $-2 \omega /\left(\pi c^{2}\right)$ times the imaginary part of the Green function $G(\mathbf{r}, \mathbf{r} ; \omega)$. In free space, all modes are propagating modes and $\rho_{0}(\mathbf{r}, \omega)=\omega^{2} /\left(2 \pi^{2} c^{3}\right)$, independent of position. In dielectric structures, the LDOS is the sum of the local density of propagating modes $\rho_{p}(\mathbf{r} ; \omega)$ and of the local density of guided modes $\rho_{g}(\mathbf{r} ; \omega)$, which we will also call the propagating and guided LDOS, respectively. Spontaneous emission can occur into both types of modes, the physical difference between the two cases being that light emitted into a propagating mode eventually can be detected in the far field, whereas light emitted into the guided modes does not leave the structure.

First we need to know the Green function of the crystal of plane scatterers. Often a Green function is given and calculated in terms of its mode decomposition, but here we proceed differently. The same $T$ matrix (20) that gave the propagating and guided modes when inserted in the LippmannSchwinger equation, also gives the Green function when inserted in the Dyson-Schwinger equation (3),

$$
\begin{aligned}
G\left(\mathbf{k}_{\|}, z, z^{\prime} ; \omega\right)= & g_{0}\left(\mathbf{k}_{\|}, z-z^{\prime} ; \omega\right)+\sum_{\alpha, \beta=1}^{N} g_{0}\left(\mathbf{k}_{\|}, z-z_{\alpha} ; \omega\right) \\
& \times T_{\alpha \beta}^{(N)}\left(\mathbf{k}_{\|} ; \omega\right) g_{0}\left(\mathbf{k}_{\|}, z_{\beta}-z^{\prime} ; \omega\right)
\end{aligned}
$$

This is the Green function in the mixed representation and in order to find the LDOS we must transform it to real space using a two-dimensional inverse Fourier transformation and then take the imaginary part. The crystal is translation invariant in the $x$ and $y$ directions so that the LDOS depends on the $z$ position only. Moreover, in our model (29) the individual planes scatter light isotropically so that also $T_{\alpha \beta}^{(N)}\left(\mathbf{k}_{\|} ; \omega\right)$ $=T_{\alpha \beta}^{(N)}\left(k_{\|} ; \omega\right)$. Then the integral over directions $\hat{k}_{\|}$is trivial; the integral over the magnitude $k_{\|}$is split into a propagating and a guided part, as we present below.

The propagating LDOS is given by

$$
\begin{aligned}
\rho_{p}(z, \omega)= & \rho_{0}(z, \omega)-\frac{\omega}{\pi^{2} c^{2}} \operatorname{Im} \int_{0}^{\omega / c} \mathrm{~d} k_{\|} k_{\|} \sum_{\alpha, \beta=1}^{N} g_{0}\left(k_{\|}, z\right. \\
& \left.-z_{\alpha} ; \omega\right) T_{\alpha \beta}^{(N)}\left(k_{\|} ; \omega\right) g_{0}\left(k_{\|}, z_{\beta}-z ; \omega\right) .
\end{aligned}
$$

The integral is taken over the magnitude of the in-plane wave vectors $k_{\|}$with values between 0 and $\omega / c$, so that all wave vectors contributing to the propagating LDOS are real.

The remaining part of the inverse Fourier transform gives the guided LDOS,

$$
\begin{aligned}
\rho_{g}(z, \omega)= & -\frac{\omega}{\pi^{2} c^{2}} \operatorname{Im} \int_{\omega / c}^{\infty} \mathrm{d} k_{\|} k_{\|} \sum_{\alpha, \beta=1}^{N} g_{0}\left(k_{\|}, z-z_{\alpha} ; \omega\right) \\
& \times T_{\alpha \beta}^{(N)}\left(k_{\|} ; \omega\right) g_{0}\left(k_{\|}, z_{\beta}-z ; \omega\right) .
\end{aligned}
$$

Here $k_{\|}>\omega / c$, so that the integrand in Eq. (40) is real except at the poles $k_{\|}(\omega)$ of the $T$-matrix elements. Now the 


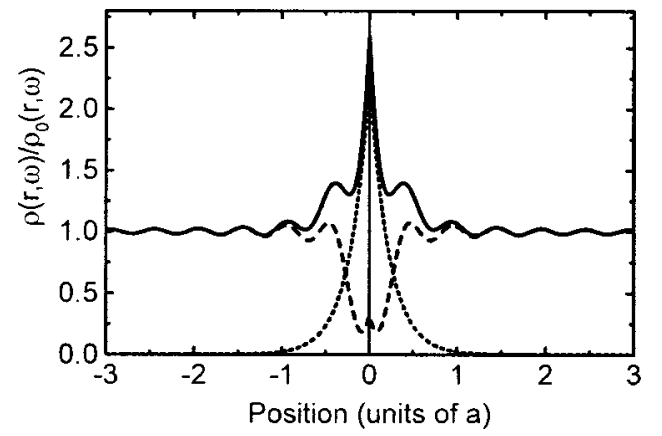

FIG. 5. Local densities of states for a single plane, relative to the free-space value, for $a / \lambda=0.5$ and $D_{\text {eff }}=0.46 a$. Both the radiative (dashed line) and the guided LDOS (dotted line) are shown, as well as their sum (solid line).

$T$-matrix elements are written in Eq. (20) in terms of the functions $h_{N}$, but it can be shown that the poles of the latter do not correspond to poles of the $T_{\alpha \beta}^{(N)}$. The $T$-matrix elements only have poles when $1+h_{N}(1,1)=0$, corresponding to the guided modes that were found in Sec. III B. The righthand side of the equality (40), therefore, simplifies into a sum over residues of the integrand at the guided-mode poles. The infinitesimally small positive imaginary parts of the poles ensure that $\rho_{g}(z, \omega) \geqslant 0$.

Now we discuss what the LDOS actually looks like in a system of plane scatterers. First consider $\rho_{p}$ for a single plane located at $z=0$. The $T$ matrix is given in Eq. (29). Exactly at the position of the plane, the relative propagating $\operatorname{LDOS} \rho_{p}(0, \omega) / \rho_{0}(0, \omega)$ turns out to be equal to the average transmission $\left\langle|\tau|^{2}\right\rangle_{\text {av }}$ through the plane [Eq. (31)]. As expected, highly reflecting planes affect $\rho_{p}$ more strongly. Figure 5 shows the local densities in the neighborhood of the plane. (Although for $N=1$ the distance between the planes $a$ is not defined, we can choose a distance $a$ and scale the plot such that $D_{\text {eff }}=0.46 a$ and $a / \lambda=0.5$.) At $z=0$ we indeed have $\rho_{p} / \rho_{0}=0.32$, equal to the average transmission of the plane. Away from the plane, $\rho_{p}$ oscillates back to $\rho_{0}$.

Figure 5 also shows the guided LDOS for the single plane at $z=0$. With the pole of the $T$ matrix located at $\kappa_{1}^{(1)}$ $=D_{\text {eff }}(\omega / c)^{2} / 2+i \eta$, the guided LDOS (40) in this case is

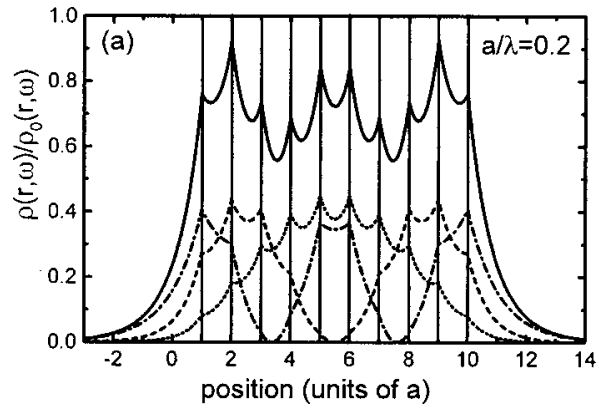

$$
\rho_{g}(z ; \omega)=\left(\frac{-2 \omega}{\pi c^{2}}\right)\left(\frac{-\kappa_{1}^{(1)}}{4}\right) \exp \left(-2 \kappa_{1}^{(1)}|z|\right) .
$$

So for $N=1$, the guided LDOS falls-off exponentially as a function of distance from the plane, with a decay length of $\left[\kappa_{1}^{(1)}(\omega)\right]^{-1}$. At the position of the plane the relative guided LDOS $\rho_{g} / \rho_{0}$ is equal to $\pi^{2} D_{\text {eff }} / \lambda$, which is equal to 2.27 for the values of the parameters of Fig. 5. So we know how at the position of the plane $\rho_{g}$ goes up and $\rho_{p}$ goes down when the effective thickness is increased.

For larger systems, for $N=10$ say, the guided modes can either be localized to individual planes or be delocalized over the entire structure. At least, this was anticipated in Sec. III B when discussing the dispersion relations of the guided modes. Now we can check this. Figures $6(a, b)$ show the propagating and guided LDOS as well as their sum, as a function of position in and around the structure, for a frequency such that $a / \lambda=0.2$. Again, the densities were divided by the free-space value for a good comparison. Local densities of individual modes are plotted in Fig. 6(a). According to Fig. 4 there are three guided modes at this frequency, with $\kappa_{1}^{(10)} a=0.84, \kappa_{2}^{(10)} a=0.77$, and $\kappa_{3}^{(10)} a=0.48$, respectively. From Fig. 6(a) one can learn that each of these three guided modes is delocalized over all the planes and also that every next guided mode has one extra node in its mode density. The guided LDOS corresponds to the solid line in Fig. 6(a); it is the sum of the contributions of the three modes and it does not behave periodically inside the crystal.

Note that $\rho_{g}(z, \omega)$ divided by the free-space value varies around 0.7 inside the structure. The guided modes are, therefore, an important channel for spontaneous emission. This is also illustrated by Fig. 6(b), where for the same frequency the guided LDOS, the radiative LDOS, as well as their sum are shown. The frequency is well below the first stop band in any direction (see Fig. 3). As is clear from Fig. 6(b), inside the structure $\rho_{p}(z, \omega)$ is smaller than $\rho_{g}(z, \omega)$. Notice also that the sum $\rho_{p}+\rho_{g}$ shows more periodicity in the structure than $\rho_{p}$ or $\rho_{g}$ separately. Outside the structure the radiative LDOS climbs back to the free-space value, showing damped oscillations; the guided LDOS drops down to zero outside the structure. The decay looks exponential with a decay length of about $\lambda / 4$. In fact, the decay is multiexponential

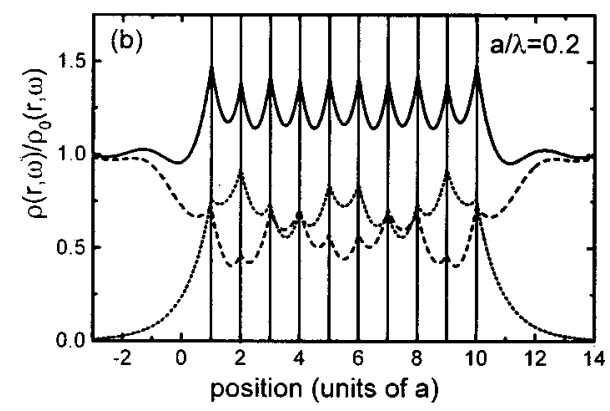

FIG. 6. Local densities of states for a crystal of plane scatterers with $N=10$; densities are divided by the free-space density. Figure (a) shows the guided mode LDOS (solid line) at $a / \lambda=0.2$. It is the sum of contributions from three guided modes: the first (dotted line) without nodes, the second one (dashed) with one node, and the third guided mode (dot dashed) with two nodes. Figure (b) shows at the same frequency the guided LDOS (dotted line), the radiative LDOS (dashed) as well as the total LDOS (solid line). 


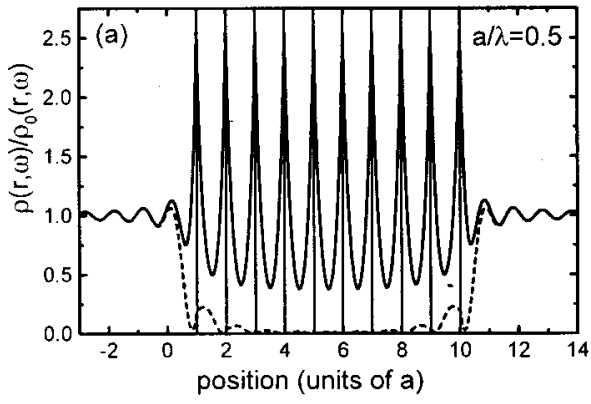

because all three guided modes decay with their own decay length. Moreover, according to Eq. (40) the exponential decay does not start at the outer planes only.

Figure 7(a) also shows local densities of states, but for a higher frequency such that $a / \lambda=0.5$. This frequency is interesting, because it is the location of the blue edge of the first stop band in normal direction, as can be seen in Fig. 3. Light that makes an angle $\theta$ with the normal has a first stop band that is shifted to higher frequencies, according to Bragg's law $2 a \cos \theta=\lambda$. At $a / \lambda=0.5$, stop bands of many directions will overlap. Therefore, it is to be expected that $\rho_{r}(\mathbf{r}, \omega)$ is much lower than in free space. Figure 7(a) shows precisely this: the $\rho_{r}(\mathbf{r}, \omega)$ rapidly drops down to almost zero inside the plane crystal. Actually, what we have in Fig. 7(a) is an omnidirectional mirror [17]. This can be checked by varying the angle of the incoming light in Eq. (32) (not shown): for all angles ranging from $0^{\circ}$ to $90^{\circ}$, the first stop bands overlap at $a / \lambda=0.5$. Figure 7 (a) suggests a working definition of a finite omnidirectional mirror: A periodic structure is an omnidirectional mirror at a frequency $\omega$ when the maximum value of $\rho_{p}(z, \omega) / \rho_{0}(z, \omega)$ in the middle unit cell is smaller than some previously agreed-upon value $\sigma \ll 1$. The parameter $\sigma$ denotes the maximum negligible amount of propagating LDOS that one accepts inside a finite omnidirectional mirror. For example, the structure of Fig. 7(a) only counts as an omnidirectional mirror when we accept a $\sigma$ of 0.013 or larger. Only for infinite structures can one find omnidirectional mirrors with $\sigma=0$. Although the number $N$ of unit cells does not explicitly enter this definition, it is clear that increasing $N$ can help to turn a structure into an omnidirectional mirror.

Figure 7(a) also shows that the guided LDOS $\rho_{g}(z, \omega)$ does not go to zero in the omnidirectional mirror. At $a / \lambda$ $=0.5$, the maximum number of ten guided modes have appeared in the ten-plane crystal. Since inside the structure the total LDOS is almost exclusively made up of $\rho_{g}(\mathbf{r}, \omega)$, spontaneous emission at this frequency occurs into one of these guided modes with almost $100 \%$ efficiency. Conversely, the extraction efficiency $\rho_{r} /\left(\rho_{g}+\rho_{r}\right)$ of light spontaneously emitted inside the crystal will be almost zero at $a / \lambda=0.5$.

Finally, in Fig. 7(b) the frequency is increased such that $a / \lambda=0.6$, above the first stop band in the normal direction (see Fig. 3). Then incident light in and around the normal direction can enter the structure again and therefore $\rho_{p}(z, \omega)$ is nonzero inside the crystal, as the figure shows. For this frequency, the structure is not an omnidirectional mirror for any reasonable tolerance parameter $\sigma$. The combined density of the ten guided modes has become even more localized to the planes, which is the only thing that happens to $\rho_{g}$ when increasing the frequency even more. Interestingly, in between the planes the radiative LDOS is small where the guided LDOS is large, and vice versa, so that the variation in the total LDOS is small. Near the planes, however, the total LDOS is much larger than the free-space value, mainly due to $\rho_{g}$.

In general, the propagating LDOS cannot be increased much beyond the free-space value, at least for the effective thickness $D_{\text {eff }}=0.46 a$ that we consider. This is somewhat surprising since individual propagating modes can be strongly resonant inside the structure (see Fig. 2). But $\rho_{p}(z, \omega)$ is an integral (39) over all directions, some of resonant and others of decaying nature. As it turns out, this does not add up to form a large increase. Even if the fraction of light spontaneously emitted into a certain direction can be dramatically increased compared to the emission in vacuum, the total emission rate into propagating modes is not increased much.

A last remark on the mirror symmetry of the LDOS in Fig. 5. The LDOS should of course be symmetrical in $z$ $=(N+1) a / 2$. In our formalism, this corresponds to a symmetry in the $T$-matrix elements, $T_{\alpha, \beta}^{(N)}=T_{N+1-\alpha, N+1-\beta}^{(N)}$. In particular, this symmetry should also be found in the residues of $T_{\alpha, \beta}^{(N)}$ at the poles $\kappa_{m}^{(N)}(\omega)$ corresponding to the guided modes. We could use the symmetry in the plots as a check on our calculations, because the plots were not made symmetrical "by hand."

\section{SUMMARY AND CONCLUSIONS}

In this paper we have used the plane scatterer as our work horse and discussed its range of validity. We put it to use in the $T$-matrix formalism of multiple-scattering theory. The $T$ matrix of $N$ identical and parallel planes located at arbitrary positions was found as the inverse of a known $N \times N$ matrix, in Eq. (18); using the trick as discussed in the Appendix, in equation (20) an explicit expression for the $T$-matrix elements of a crystal of regularly spaced plane scatterers was given.

These analytical results enabled us to find relatively easily both the propagating modes [in Eq. (32)] and the guided modes [defined by Eq. (35)] of a crystal consisting of an arbitrary but finite number of planes. Not only individual modes, but also the complete Green function could be found [Eq. (38)]. From the Green function, the local density of states can be calculated, which is the sum of a propagating and a guided LDOS. The sum of the two determines the local 
spontaneous emission rate and the relative contribution of the propagating to the total LDOS gives you the extraction efficiency of spontaneously emitted light. Clearly, in order to make a connection with experiments, it is important to know the Green function of finite crystals. The knowledge of the Green function and the LDOS is also needed for the interpretation of images recorded with a scanning near-field optical microscope [35]. Here especially the LDOS near the surface of the crystal is important.

We chose to present results for $N=10$. The ten-plane crystal was shown to exhibit stop bands. Around the frequencies of the first stop band in normal direction, guided modes appear as delocalized over the whole crystal whereas for higher frequencies they localize to the individual planes. Around $a / \lambda=0.5$ the crystal is an omnidirectional mirror. We argued to consider a finite periodic structure as an omnidirectional mirror if the maximal value of the propagating LDOS in its innermost unit cell is smaller than some previously agreed-upon finite value. The LDOS of the crystal of planes is a well-behaved function of position and frequency. The variations inside a unit cell can be large. Evidently, the finite translation symmetry of the LDOS is broken for the finite crystals, especially near the surfaces. The total LDOS shows more translation symmetry than the propagating or guided LDOS separately, which can be seen as a competition between both types of modes with different outcomes when going from one unit cell to another.

\section{ACKNOWLEDGMENTS}

We would like to thank Rudolf Sprik, Leendert Suttorp, and Willem Vos for stimulating discussions. This work is part of the research program of the Stichting voor Fundamenteel Onderzoek der Materie, which is financially supported by the Nederlandse Organisatie voor Wetenschappelijk Onderzoek.

\section{APPENDIX: ANALYTICAL EXPRESSION FOR $T$ MATRIX OF $N$-PLANE CRYSTAL}

The problem that arose in Sec. II B and that is solved in this appendix is how to find the inverse of the matrix

$$
\mathbf{M} \equiv\left(\begin{array}{cccc}
\mu & x & x^{2} & x^{3} \\
x & \mu & x & x^{2} \\
x^{2} & x & \mu & x \\
x^{3} & x^{2} & x & \mu
\end{array}\right)
$$

The solution will be for general $N$, but matrices are presented for $N=4$. Define the upper diagonal matrix $\mathbf{U}$ and lower diagonal matrix $\mathbf{L}$ as

$$
\mathbf{U}=\left(\begin{array}{llll}
0 & x & 0 & 0 \\
0 & 0 & x & 0 \\
0 & 0 & 0 & x \\
0 & 0 & 0 & 0
\end{array}\right) ; \quad \mathbf{L}=\left(\begin{array}{cccc}
0 & 0 & 0 & 0 \\
x & 0 & 0 & 0 \\
0 & x & 0 & 0 \\
0 & 0 & x & 0
\end{array}\right)
$$

Then we have

$$
\mathbf{U}^{2}=\left(\begin{array}{cccc}
0 & 0 & x^{2} & 0 \\
0 & 0 & 0 & x^{2} \\
0 & 0 & 0 & 0 \\
0 & 0 & 0 & 0
\end{array}\right) ; \quad \mathbf{U}^{N}=0
$$

and similarly for powers of the lower diagonal matrix $\mathbf{L}$. From this, it follows that we can write the matrix $\mathbf{M}$ as

$$
\mathbf{M}=\mu \rrbracket+\sum_{p=1}^{N-1}\left(\mathbf{U}^{p}+\mathbf{L}^{p}\right)=\mu \rrbracket+\mathbf{U}(\mathbb{1}-\mathbf{U})^{-1}+(\mathbb{1}-\mathbf{L})^{-1} \mathbf{L} .
$$

Now consider the inverse of the matrix product $(1-\mathbf{L}) \mathbf{M}(1$ $-\mathbf{U}$ ). Use this and Eq. (A4) to arrive at the following expression for the inverse of $\mathbf{M}$ :

$$
\begin{aligned}
\mathbf{M}^{-1}= & (\mathbb{1}-\mathbf{U})[\mu(\mathbb{1}-\mathbf{L})(\mathbb{1}-\mathbf{U})+(\mathbb{1}-\mathbf{L}) \mathbf{U} \\
& +\mathbf{L}(\mathbb{1}-\mathbf{U})]^{-1}(\mathbb{1}-\mathbf{L}) .
\end{aligned}
$$

In this form, finding the inverse is much easier than before, because the matrix $[\cdots]$ has only nonzero elements on the diagonal and first off diagonals. This becomes clearer by rewriting

$$
\begin{aligned}
\mathbf{M}^{-1} & =(\mathbb{1}-\mathbf{U})[\mathbf{Y}+\mathbf{Z}]^{-1}(\mathbb{1}-\mathbf{L}) \\
& =(\mathbb{1}-\mathbf{U})\left[1+\mathbf{Y}^{-1} \mathbf{Z}\right]^{-1} \mathbf{Y}^{-1}(\mathbb{1}-\mathbf{L}),
\end{aligned}
$$

where $\mathbf{Y} \equiv\left(\mu+\mu x^{2}-2 x^{2}\right) 1+(1-\mu)(\mathbf{U}+\mathbf{L})$ is a symmetric band diagonal matrix and the matrix $\mathbf{Z}$ has matrix elements $Z_{k l} \equiv(2-\mu) x^{2} \delta_{k 1} \delta_{l 1}$ so that only its upper left element is nonzero.

The matrix $\mathbf{M}^{-1}$ is known when $\mathbf{Y}^{-1}$ and $\left[1+\mathbf{Y}^{-1} \mathbf{Z}\right]^{-1}$ are known. Now $\mathbf{Y}$ has a simple structure and it can be inverted immediately using the transformation matrix $J_{k l}$ $\equiv \sqrt{2 /(N+1)} \sin [k l \pi /(N+1)]$. It is its own inverse and it diagonalizes $\mathbf{Y}$ : we have $\mathbf{Y}^{-1}=\mathbf{J} \boldsymbol{\Lambda}^{-1} \mathbf{J}$ with $\Lambda_{k l}^{-1}=\delta_{k l} f^{-1}(k)$ where the function $f(k)$ is defined as

$$
\begin{aligned}
f(k) \equiv & \mu\left(1+x^{2}\right)-2 x^{2}+2 x(1-\mu) \cos [k \pi /(N+1)] \\
& (k=1,2, \ldots, N) .
\end{aligned}
$$

What remains to be done for the inversion of $\mathbf{M}$ is the evaluation of the infinite series

$$
\left(\mathbb{1}+\mathbf{Y}^{-1} \mathbf{Z}\right)^{-1}=\mathbb{1}-\mathbf{Y}^{-1} \mathbf{Z}+\left(\mathbf{Y}^{-1} \mathbf{Z}\right)^{2}-\left(\mathbf{Y}^{-1} \mathbf{Z}\right)^{3}+\cdots .
$$

Now since we have

$$
\left(Y^{-1} Z\right)_{k p}=(2-\mu) x^{2} \delta_{1 p} \sum_{m=1}^{N} J_{k m} f^{-1}(m) J_{m p} \equiv h_{N}(k, p) \delta_{1 p},
$$


where in the last equality the function $h_{N}(k, p)$ was defined, we find that $\left[\left(\mathbf{Y}^{-1} \mathbf{Z}\right)^{n}\right]_{k p}=h_{N}(k, 1) h_{N}^{n-1}(1,1) \delta_{1 p}$. Summing up all orders, we find that

$$
\left(1+Y^{-1} Z\right)_{k p}^{-1}=\left[\delta_{k p}-\left(\frac{h_{N}(k, 1)}{1+h_{N}(1,1)}\right) \delta_{1 p}\right] .
$$

Inserting this into Eq. (A6), we have found the inverse of the matrix M. This result is used in Eq. (20) of Sec. II B.

For Sec. III B it is useful to compute the determinant of the matrix $\mathbf{M}$ and this can be done best using Eq. (A6), $\operatorname{det}(\mathbf{M})=\operatorname{det}\left[\mathbf{Y}\left(\mathbb{1}+\mathbf{Y}^{-1} \mathbf{Z}\right)\right]$. This determinant is equal to $\left[1+h_{N}(1,1)\right]$ times the product of all the $f(k)$ 's of Eq. (A7). With Eq. (22) and the definition of the Bloch wave vector this gives

$$
\begin{aligned}
\operatorname{det}\left[\mathbf{M}\left(k_{\|} ; \omega\right)\right]= & {\left[\frac{-1}{2 x(1-\mu)}\right]^{N}\{\sin [(N+1) K a]} \\
& \left.-\left[\frac{(2-\mu) x}{1-\mu}\right] \sin [N K a]\right\} \\
& \times \frac{\prod_{m=1}^{N}\left[\cos (K a)-\cos \left(\frac{m \pi}{N+1}\right)\right]}{\sin [(N+1) K a]} .
\end{aligned}
$$

Notice that the zeroes of the determinant are not caused by the products of $\cos (K a)-\cos ([m \pi / N+1])$ going to zero, because of the $\sin [(N+1) K a]$ in the denominator. The determinant is zero when of the other factor in the nominator is zero, which is equivalent to $\left[1+h_{N}(1,1)\right]=0$.
[1] E. Yablonovitch, Phys. Rev. Lett. 58, 2059 (1987); S. John, ibid. 58, 2486 (1987).

[2] M. S. Thijssen, R. Sprik, J. E. G. J. Wijnhoven, M. Megens, T. Narayanan, A. Lagendijk, and W. L. Vos, Phys. Rev. Lett. 83, 2730 (1999).

[3] A. Blanco, E. Chomski, S. Grabtchak, M. Ibisate, S. John, S. W. Leonard, C. Lopez, F. Meseguer, H. Miguez, J. P. Mondia, G. A. Ozin, O. Toader, and H. M. van Driel, Nature (London) 405, 437 (2000).

[4] A. Figotin, in Diffusive Waves in Complex Media, edited by J. P. Fouque (Kluwer, Dordrecht, 1999).

[5] A. Tip, A. Moroz, and J. M. Combes, J. Phys. A 33, 6223 (2000).

[6] R. Sprik, B. A. van Tiggelen, and A. Lagendijk, Europhys. Lett. 35, 265 (1996).

[7] K. Busch and S. John, Phys. Rev. E 58, 3896 (1998).

[8] A. A. Asatryan, K. Busch, R. C. McPhedran, L. C. Botten, C. M. de Sterke, and N. A. Nicorovici, Phys. Rev. E 63, 046612 (2001); A. A. Asatryan, S. Fabre, K. Busch, R. C. McPedran, L. C. Botten, C. M. de Sterke, N. A. Nicorovici, Opt. Express 8, 191 (2001).

[9] M. J. A. de Dood, L. H. Slooff, A. Polman, A. Moroz, and A. van Blaaderen, Phys. Rev. A 64, 033807 (2001).

[10] T. Suzuki and P. K. L. Yu, J. Opt. Soc. Am. B 12, 570 (1995).

[11] Z.-Y. Li and Y. Xia, Phys. Rev. A 63, 043817 (2001).

[12] M. Born and E. Wolf, Principles of Optics (Cambridge University Press, Cambridge, UK, 1999).

[13] A. Yariv and P. Yeh, Optical Waves in Crystals (Wiley, New York, 1984).

[14] M. D. Tocci, M. Scalora, M. J. Bloemer, J. P. Dowling, and C. M. Bowden, Phys. Rev. A 53, 2799 (1996).

[15] H. Rigneault, S. Maure, and C. Amra, Pure Appl. Opt. 7, 549 (1998).

[16] H. Rigneault and S. Monneret, Phys. Rev. A 54, 2356 (1996).

[17] Y. Fink, J. N. Winn, S. Fan, C. Chen, J. Michel, J. D.
Joannopoulos, and E. L. Thomas, Science 282, 1679 (1998).

[18] C. Hooijer, D. Lenstra, and A. Lagendijk, Opt. Lett. 25, 1666 (2000).

[19] J. M. Bendickson, J. P. Dowling, and M. Scalora, Phys. Rev. E 53, 4107 (1996).

[20] M. S. Tomaš, Phys. Rev. A 51, 2545 (1995).

[21] R. Petit and G. Tayeb, J. Opt. Soc. Am. A 7, 1686 (1990).

[22] J. P. Dowling and C. M. Bowden, Phys. Rev. A 46, 612 (1992).

[23] T. J. Shepherd, P. J. Roberts, and R. Loudon, Phys. Rev. E 55, 6024 (1997).

[24] A. Messiah, Quantum Mechanics (North-Holland, Amsterdam, 1962), Vol. II, Chap. 19.

[25] R. G. Newton, Scattering Theory of Waves and Particles (McGraw-Hill, New York, 1966).

[26] A. Gonis and W. H. Butler, Multiple Scattering in Solids (Springer, New York, 2000).

[27] A. Lagendijk and B. A. van Tiggelen, Phys. Rep. 270, 146 (1996).

[28] P. de Vries, D. V. van Coevorden, and A. Lagendijk, Rev. Mod. Phys. 70, 447 (1998).

[29] D. V. van Coevorden, R. Sprik, A. Tip, and A. Lagendijk, Phys. Rev. Lett. 77, 2412 (1996).

[30] E. N. Economou, Green's Functions in Quantum Physics (Springer, Berlin, 1979).

[31] L. Mandel and E. Wolf, Optical Coherence and Quantum Optics (Cambridge University Press, Cambridge, UK, 1995).

[32] E. R. Hansen, A Table of Series and Products (Prentice-Hall, Englewood Cliffs, NJ, 1975), Eq. (41.2.19).

[33] J. D. Jackson, Classical Electrodynamics (Wiley, New York, 1975).

[34] J. E. G. J. Wijnhoven and W. L. Vos, Science 281, 802 (1998).

[35] G. Colas des Francs, C. Girard, J.-C. Weeber, C. Chicane, T. David, A. Dereux, and D. Peyrade, Phys. Rev. Lett. 86, 4950 (2001); G. Colas des Francs, C. Girard, J.-C. Weeber, and A. Dereux, Chem. Phys. Lett. 345, 512 (2001). 